Oct 2, 1995

IASSNS-HEP-95/76

hep-th/9509176

\title{
On the Quantum Moduli Space of Vacua of $N=2$ Supersymmetric Gauge Theories
}

\author{
Amihay Hanany \\ hanany@sns.ias.edu \\ School of Natural Sciences \\ Institute for Advanced Study \\ Olden Lane, Princeton, NJ 08540, USA
}

\begin{abstract}
Families of hyper-elliptic curves which describe the quantum moduli spaces of vacua of $N=2$ supersymmetric $S O\left(N_{c}\right)$ gauge theories coupled to $N_{f}$ flavors of quarks in the vector representation are constructed. The quantum moduli spaces for $2 N_{f}<N_{c}-1$ are determined completely by imposing $R$ symmetry, instanton corrections and the proper classical singularity structure. These curves are verified by residue calculations. The quantum moduli spaces for $2 N_{f} \geq N_{c}-1$ theories are parameterized and their general structure is worked out using residue calculations. The exact metrics on the quantum moduli spaces as well as the exact spectrum of stable massive states are derived. The results presented here together with recent results of Martinec and Warner provide a natural conjecture for the form of the curves for the other gauge groups.
\end{abstract}




\section{Introduction}

There has been much progress recently in the study of non-perturbative properties of $N=2$ four dimensional supersymmetric gauge theories. Seiberg and Witten [1] found an exact soultion of the low energy effective action as well as discovered a reach structure of duality phenomena. This work was generalized in [2] to include massless and massive matter hyper-multiplets. The quantum moduli space of $N=2$ supersymmetric pure $S U\left(N_{c}\right)$ gauge theories was constructed as an $N_{c}-1$ parameter family of hyper-elliptic

curves in $[3,4]$, and the physics of the model was studied in $[5,6]$. The inclusion of matter multiplets was carried out in $[7,8]$ and was also considered in [9]. The curves for $S O(2 l+1)$ gauge groups were constructed in [10] and for $S O(2 l)$ gauge groups in [11].

Our aim in this paper is to determine the quantum moduli spaces of vacua of the Coulomb phase of $N=2$ supersymmetric $S O\left(N_{c}\right)$ gauge theories with $N_{f}$ matter hypermultiplets in the vector representation, as well as the exact metric on the quantum moduli space and the exact spectrum of massive stable particles. The quantum moduli spaces will be constructed as families of hyper-elliptic curves satisfying a set of physical constraints: $R$-symmetry, singularity structure and global symmetries, appropriate inclusion of instanton correction, proper classical and scaling (integration of massive quarks) limits, compatibility of residue calculations with the BPS formula and correct weak coupling monodromies.

The paper is organized as follows. In section two we review general aspects of $N=2$ gauge theories and the moduli spaces of vacua, introduce the physical quantities associated with them, discuss their singularity structure and present principles for their construction. In section three we construct the quantum moduli space for $N_{f}<N_{c}-l-2$. We show that it is determined completely by imposing $R$-symmetry, instanton corrections and the proper classical singularity structure. We get compatibility of the residue calculations with the BPS formula which provides a consistency check of the result. The begining of section four is devoted to the $N_{f}=N_{c}-l-2$ theories. Applying the physical constraints including residue calculations leaves us with one undetermined constant parameter.

In the rest of section four the quantum moduli spaces for $N_{f}>N_{c}-l-2$ theories are parameterized and their general structure is worked out using residue calculations. This still leaves us with undetermined constant coefficients. Global symmetry considerations may suggest a complete determination of them. We absorb the factors into redefinition of the symmetric polynomials. Weak coupling monodromy calculations along the lines of [7] give support to the results in the previous sections. 
In section five we discuss the $N_{f}=N_{c}-2$ theories. When the bare masses and all but one of the moduli are zero one expects scale invariant theories with periods satisfying the classical relations. The quantum moduli spaces in the massless as well as the massive theories are parameterized and their general structure is worked out using residue calculations in a similar manner to the $N_{f}>N_{c}-l-2$ case. Again, global symmetry considerations may suggest a complete description but we do not find such yet. We absorb them into redefinition of the moduli.

In general our results provide the exact metrics on the quantum moduli spaces as well as the exact spectrum of stable massive states. Section six is devoted to discussion and conclusions. We outline there a conjecture for the curves to any simple group.

\section{The moduli space of vacua of $N=2$ gauge theories}

In this section we review general aspects of $N=2$ gauge theories and the moduli spaces of vacua, introduce the physical quantities associated with them, discuss their singularity and monodromy structures, and present principles for their construction.

\section{1 $N=2$ QCD and the moduli space of vacua}

We will consider $N=2$ supersymmetric gauge theories with gauge group $G=S O\left(N_{c}\right)$ and $N_{f}$ flavors. The field content of the theories consists of $N=2$ chiral multiplet and $N_{f}$ hyper-multiplets. The $N=2$ chiral multiplet contains gauge fields $A_{\mu}$, two Weyl fermions $\lambda$ and $\psi$ (alternatively one Dirac fermion) and a complex scalar $\phi$, all in the adjoint representation of the gauge group G. The $N=2$ hyper-multiplets that we will consider contain two Weyl fermions $\psi_{q}$ and $\psi_{\tilde{q}}^{\dagger}$ and two complex bosons $q$ and $\tilde{q}^{\dagger}$, all in the vector representation $r$ of $G$.

In terms of $N=1$ super-fields the $N=2$ chiral multiplet consists of a vector multiplet $W_{\alpha}$ and a chiral multiplet $\Phi$, while the $N=2$ hyper-multiplets consist of two chiral superfields $Q^{i}{ }_{a}$ and $\tilde{Q}_{i a}$, where $i=1, \ldots, N_{f}$ is the flavor index and $a=1, \ldots, N_{c}$ is the color index. The super-potential in the $N=1$ language reads

$$
W=\sqrt{2} \tilde{Q}_{i} \Phi Q^{i}+\sum_{i} m_{i} \tilde{Q}_{i} Q^{i}
$$

with $m_{i}$ being the bare masses and color indices are suppressed. The first term in (2.1) is related by $N=2$ super-symmetry to the gauge couplings and the second term corresponds to $N=2$ invariant mass terms. 
The theory has an $l$ complex dimensional moduli space of vacua, which are parameterized by the gauge invariant order parameters

$$
u_{2 k}=\operatorname{Tr}\left\langle\phi^{2 k}\right\rangle, \quad k=1, \ldots, l,
$$

$\phi$ being the scalar field of the $N=2$ chiral multiplet. $l=\frac{N_{c}}{2}$ for $N_{c}$ even and $l=\frac{N_{c}-1}{2}$ for $N_{c}$ odd. The moduli space of vacua corresponds to $\phi$ satisfying a D-flatness condition $\left[\phi, \phi^{\dagger}\right]=0$. Thus, up to a gauge transformation we can take $\phi$ to be a block diagonal matrix,

$$
\langle\phi\rangle=\operatorname{diag}\left[A_{1}, \ldots, A_{l}\right]
$$

where $A_{i}=\left(\begin{array}{cc}0 & a_{i} \\ -a_{i} & 0\end{array}\right)$. At weak coupling we have

$$
u_{2 k}=\sum_{i=1}^{l} a_{i}^{2 k}
$$

Alternative gauge invariant order parameters are defined at the classical level as the symmetric polynomials $s_{2 k}$ in $a_{i}^{2}$

$$
s_{2 k}=(-)^{k} \sum_{i_{1}<\cdots<i_{k}} a_{i_{1}}^{2} \cdots a_{i_{k}}^{2}, \quad k=1, \ldots, l
$$

Using (2.4) and (2.5) the sets $s_{k}$ and $u_{k}$ are related by Newton's formula

$$
k s_{k}+\sum_{i=1}^{k} s_{k-i} u_{i}=0, \quad k=\{1,2, \ldots\},
$$

where $s_{0}=1, s_{2 k+1}=u_{2 k+1}=0$. This relation serves as a definition of $s_{k}$ at the quantum level.

At a generic point the expectation values of $\phi$ break the gauge symmetry to $U(1)^{l}$ and a low-energy effective Lagrangian can be written in terms of multiplets $\left(A_{i}, W_{i}\right)$, $i=1, \ldots, l$.

The $N=2$ effective Lagrangian takes the form

$$
\mathcal{L}_{e f f}=\operatorname{Im} \frac{1}{4 \pi}\left[\int d^{4} \theta \partial_{i} \mathcal{F}(A) \bar{A}^{i}+\frac{1}{2} \int d^{2} \theta \partial_{i} \partial_{j} \mathcal{F}(A) W^{i} W^{j}\right]
$$

where $\mathcal{F}$ is a holomorphic pre-potential.

$N=2$ super-symmetry implies that there are no perturbative corrections beyond one loop. There are, however, non-perturbative instanton corrections. 
The classical moduli space is described by the family of genus $g=2 l-1$ hyper-elliptic curves given by $[10,11]$

$$
y^{2}=\mathcal{C}^{2}(x), \quad \mathcal{C}(x)=\operatorname{det}\left(x I_{c}-\phi\right),
$$

where $I_{c}$ is the $N_{c} \times N_{c}$ identity matrix and in terms of the symmetric functions $s_{i}$

$$
\mathcal{C}(x)=x^{2 l}+\sum_{i=1}^{l} s_{2 i} x^{2(l-i)}
$$

The singularities of the classical curve correspond to cases where the gauge group is spontaneously broken to non-Abelian subgroups of $G$ rather than to $U(1)^{l}$.

We will construct the quantum moduli spaces of vacua for the $S O\left(N_{c}\right)$ groups (For other groups we will use a description recently advocated by Martinec and Warner [17].) as families of hyper-elliptic curves

$$
y^{2}=\prod_{i=1}^{4 l}\left(x-e_{i}\right)
$$

where the roots $e_{i}$ are functions of the quark masses $m_{i}$, the dynamically generated scale $\Lambda{ }^{*}$ and the gauge invariant order parameters $u_{k}$ or $s_{k}$, with (2.8) being their classical limit.

\subsection{Dyon spectrum and duality}

The spectrum of $N=2$ supersymmetric QCD includes particles in the "small" representation of the $N=2$ algebra, the so called BPS-saturated states. These are electrically and magnetically charged particles with masses

$$
M^{2}=2|Z|^{2}
$$

where $Z$ is a central extension of the $N=2$ supersymmetry algebra and is a linear combination of conserved charges [12]. It reads [2]

$$
Z=\sum_{i=1}^{l}\left[n_{e}^{i} a^{i}+n_{m}^{i} a_{D}^{i}\right]+\sum_{i=1}^{N_{f}} S_{i} m_{i}
$$

where $n_{e}, n_{m}$ are the electric and magnetic charges. $S_{i}$ are the $U(1)$ charges corresponding to additional symmetries that may exist when the global symmetry is explicitly broken

\footnotetext{
${ }^{*}$ When $2 h=2 N_{f} \mu$, the role of the dynamical scale $\Lambda$ is played by a modular form of the appropriate modular group. $h$ is the second Casimir and $\mu$ is the Dynkin index of the representation. For $S O\left(N_{c}\right)$ $h=N_{c}-2, \mu=1$
} 
by non-zero masses. $m_{i}$ are the bare masses of the hyper-multiplets and $a, a_{D}$ correspond to the vacuum expectation values of the scalar component of the chiral super-field $A$ and its dual

$$
a_{D}^{i}=\frac{\partial \mathcal{F}(a)}{\partial a} .
$$

Mathematically, $a, a_{D}$ are sections of a certain bundle over the moduli space of vacua. When all the masses $m_{i}$ are zero, $a, a_{D}$ are sections of an $S p(2 l, Z)$ bundle, thus their monodromies upon traversing a closed cycle in the moduli space are elements of $S p(2 l, Z)$. The mass formula (2.11) is $S p(2 l, Z)$ invariant, reflecting a duality property of the theory. When the masses do not vanish, constant shifts are allowed transformations in addition to the above $S p(2 l, Z)$ structure $[1,2]$. Thus, the monodromy transformation takes the form

$$
\left(\begin{array}{c}
a_{D} \\
a
\end{array}\right) \rightarrow M\left(\begin{array}{c}
a_{D} \\
a
\end{array}\right)+c, \quad M \in S p(2 l, Z),
$$

where $c$ is independent of the moduli parameters and depends on the masses of the quarks.

$a, a_{D}$ can be written as periods of a meromorphic one form $\lambda$ on the curve describing the space of vacua

$$
a_{D}^{i}=\oint_{\alpha_{i}} \lambda, \quad a^{i}=\oint_{\beta_{i}} \lambda,
$$

where $\alpha_{i}, \beta_{j}$ form a basis of homology cycles on the curve. In order to determine $\lambda$ one assumes that

$$
\begin{aligned}
\frac{\partial a_{D}^{i}}{\partial s_{2 k}} & \propto \oint_{\alpha_{i}} x^{2(l-k-1)} \frac{d x}{y} \quad k=1, \ldots, l \\
\frac{\partial a^{i}}{\partial s_{2 k}} & \propto \oint_{\beta_{i}} x^{2(l-k-1)} \frac{d x}{y} \quad k=1, \ldots, l,
\end{aligned}
$$

with $x^{2(l-k-1)} \frac{d x}{y} \quad k=1, \ldots, l$ being a basis of holomorphic one forms on the curve. The exact proportionality factor in (2.16) is determined by matching $a^{i}$ to the weak coupling relations (2.4) or (2.5). It is a constant since we want to avoid unwanted zeros or poles. Its value is $\frac{1}{2 \pi i}$.

Note that (2.15) and (2.16) yield, up to an exact form,

$$
\frac{d \lambda}{d s_{k}} \propto x^{2(l-k-1)} \frac{d x}{y} \quad k=1, \ldots, l .
$$

When the bare masses are zero the residues of $\lambda$ vanish, thus ensuring that $a, a_{D}$ are invariant under deformations of the cycle of integration even across poles of $\lambda$. For non zero masses the residues take the form

$$
2 \pi i \operatorname{res}(\lambda)=\sum_{i} n_{i} m_{i} \quad n_{i} \in Z,
$$


thus allowing jumps in $a, a_{D}$, as defined in (2.15), when crossing poles of $\lambda$ compatible with the mass formula (2.12).

The matrix of low energy coupling constants, $\tau$, is given by

$$
\tau_{i j}(a)=\frac{\partial a_{D}^{i}}{\partial a^{j}} .
$$

By virtue of (2.16), $\tau_{i j}$ is the period matrix of the curve describing the quantum moduli space. The metric on quantum moduli space reads

$$
(d s)^{2}=\operatorname{Im} d a_{D}^{i} d \bar{a}^{i}
$$

is invariant under the transformation (2.14), and is positive definite.

\subsection{The singularity structure}

As discussed in section 2.1, the $l$ dimensional moduli space of vacua $\mathcal{M}_{l}$ is parameterized by gauge invariant order parameters such as $s_{k}$ of equation (2.5). The singular locus of the family of curves (2.10) describing the moduli space is the codimension one variety defined as the vanishing locus of the discriminant*

$$
\Delta\left[s_{k}\right] \equiv \prod_{i<j}\left(e_{i}-e_{j}\right)^{2}=0 .
$$

Along this variety additional massless states appear in the spectrum and the effective low energy description (2.7) is not valid.

In the semi-classical limit $\Lambda \rightarrow 0$ the discriminant factorizes

$$
\Delta[\Lambda \rightarrow 0]=\Lambda^{h_{g}\left(2 h-2 N_{f} \mu\right)} \Delta_{c}^{2} \Delta_{f},
$$

with $h_{g}=2 l$ for $S O\left(N_{c}\right)$. where

$$
\Delta_{c}=\Delta(\mathcal{C})
$$

is the discriminant of $\operatorname{calC}(x)$ and

$$
\Delta_{f}=\prod_{j=1}^{N_{f}} \mathcal{C}^{2 \mu}\left(x^{2}=m_{i}^{2}\right) .
$$

The zero locus of $\Delta_{c}$ corresponds to singularities where classically the group $G$ is not spontaneously broken to $U(1)^{l}$ but rather to a larger subgroup (In particular, the gauge

${ }^{*}$ When the coefficient of the highest order monomial $x^{n}$ in the polynomial is $a \neq 1$ the discriminant (2.21) is modified by a pre-factor $a^{2 n-2}$. 
group is not broken at all at the origin of the moduli space). The zero locus of $\Delta_{f}$ defines a complex codimension one variety in the moduli space where a quark becomes massless classically. The product up to $N_{f}$ in (2.24) is a consequence of having $N_{f}$ different flavors. The power of $\Lambda$ in (2.22) follows from instanton contribution and from the fact that classically $N_{c}$ roots degenerate.

\subsection{Principles for the construction of the moduli spaces}

In the following we summarize the principles which will be used by us in the construction of the families of hyper-elliptic curves describing the quantum moduli spaces of vacua:

1. Symmetry: The curves are invariant under $R$ charge transformation

$$
O \rightarrow \exp \left[\frac{2 \pi i R(O)}{\left.4\left(2 h-2 N_{f} \mu\right)\right)}\right] O,
$$

where $R(O)$ is the $R$ charge of $O$ and $O$ refers to the building blocks of the curves, $y, x, m_{i}, \Lambda, s_{k}$. We have

\begin{tabular}{|c||c|c|c|c|c|c|}
\hline$O$ & $y$ & $x$ & $m_{i}$ & $\Lambda$ & $u_{k}$ & $s_{k}$ \\
\hline$R(O)$ & $4 l$ & 2 & 2 & 2 & $2 k$ & $2 k$ \\
\hline
\end{tabular}

2. Singularity structure : As discussed in section 2.3, singularities of a curve describing a quantum moduli space are associated with the zero locus of the corresponding discriminant and are physically interpreted as the appearance of additional massless states in the spectrum, where the order of vanishing of the discriminant at a point corresponding to a singularity of the curve is generically the number of massless states at that point. These states belong to a representation of the global symmetry group and the order of vanishing of the discriminant is its dimension. Thus, global symmetry imposes constraints on the singularity structure.

3. Instanton corrections : The one instanton process contribution to the curves for $2 N_{f} \mu<2 h$ takes the form [13]

$$
\Lambda^{2 h-2 N_{f} \mu} .
$$


4. Integration of a massive quark: Sending a quark mass $m \rightarrow \infty$ in $N_{f} \mu<h$ theories, and sending the scale $\Lambda_{N_{f}} \rightarrow 0$ such that *

$$
\Lambda_{N_{f}-1}^{2 h-2\left(N_{f}-1\right) \mu}=m^{2 \mu} \Lambda_{N_{f}}^{2 h-2 N_{f} \mu},
$$

is fixed, we reduce the number of flavors from $N_{f}$ to $N_{f}-1$ and we require compatibility of the curves. When $2 N_{f} \mu=2 h$ the appropriate matching condition reads

$$
\Lambda_{2 h-1}^{2 \mu}=m^{2 \mu} q,
$$

where $q \equiv e^{2 \pi i \tau}$.

5. Classical limit: The curves should exhibit the singularity structure (2.22)-(2.24) at the classical level $\Lambda \rightarrow 0$.

6. Residues : As discussed in section 2.2, the meromorphic one form on the curve $\lambda$ in (2.15) may have poles but its residues are restricted by (2.18). This, as we shall see, leads to powerful constraints on the structure of the curves.

Our aim is to construct the hyper-elliptic curves describing the quantum moduli spaces of $N=2$ QCD with $N_{c}$ colors and $N_{f}$ flavors in the vector representation of the gauge group. For this, the form of the hyper-elliptic curves introduced in $[10,11]$ is the appropriate framework. The general strategy for constructing the curves will be to first restrict the possible terms to those compatible with $R$ charge symmetry and the form of the instanton corrections. We then impose the proper classical singularity structure and use residue calculations.

Since the one loop beta function of the theory is proportional to $2 h-2 N_{f} \mu$ (higher loop corrections to it vanish) we limit ourselves to $N_{f} \mu<h$ where the theory is asymptotically free and to $N_{f} \mu=h$ where the (massless) theory is scale invariant.

\section{The quantum moduli space for $N_{f} \mu<h-l$}

\subsection{The general case}

For $N_{f} \mu<h-l$ there exist flat directions along which the $S O\left(N_{c}\right)$ gauge group is generically broken to $U(1)^{l}$ and the theory is in a Coulomb phase: There is a collection of $l$ massless photons in the spectrum. In these regions, as we shall argue, $R$-symmetry,

${ }^{*}$ In [14] this renormalization scheme was identified as the $\overline{D R}$ scheme. 
classical singularity and the form of the instanton corrections are sufficient in order to fully determine the quantum moduli spaces. First recall the curve for $N_{f}=0$. It reads $[10,11]$

$$
\begin{aligned}
& y^{2}=\mathcal{C}^{2}(x)-\Lambda_{0}^{2\left(N_{c}-2\right)} x^{2} \quad N_{c} \text { odd }, \\
& y^{2}=\mathcal{C}^{2}(x)-\Lambda_{0}^{2\left(N_{c}-2\right)} x^{4}
\end{aligned}
$$

where $\mathcal{C}(x)$ is given by $(2.9)$.

When $N_{f}$ flavors in the vector representation of the gauge group are present such that $N_{f}<h-l$ we have the following claim:

Claim: The curve describing the quantum moduli space with $N_{f}<N_{c}-l-2$ flavors is:

$$
\begin{aligned}
& y^{2}=\mathcal{C}^{2}(x)-\Lambda_{N_{f}}^{2\left(N_{c}-2-N_{f}\right)} x^{2} \operatorname{det}\left(x^{2} I_{f}-M^{2}\right) \\
& y^{2}=\mathcal{C}^{2}(x)-\Lambda_{N_{f}}^{2\left(N_{c}-2-N_{f}\right)} x^{4} \operatorname{det}\left(x^{2} I_{f}-M^{2}\right)
\end{aligned}
$$

where $I_{f}$ is the $N_{f} \times N_{f}$ identity matrix. Henceforth we will denote $d=2$ for odd $N_{c}$ and $d=4$ for even $N_{c}$. $M$ is the bare mass matrix of the quarks. Proof: $R$-symmetry, the form of instanton corrections together with the classical singularity of the gauge group imply that the most general curve is

$$
y^{2}=\mathcal{C}(x)^{2}-\Lambda_{N_{f}}^{2 h-2 N_{f} \mu} G\left(x, m_{i}\right) \equiv P,
$$

where $G\left(x, m_{i}\right)$ is a polynomial of degree $2 N_{f} \mu$ in $x$. A priori, $G$ may also depend on the moduli $s_{k}$. In the sequel it will be shown that in fact it is independent of the moduli. The second term in (3.3) is the quantum correction to the classical curve (2.8). Note that there are only one instanton contributions to the curve (3.3) due to the $R$ charge restriction. The polynomial $G\left(x, m_{i}\right)$ is determined by requiring that the discriminant of the polynomial $P$ in (3.3) has the right classical limit (2.22). In order to do this we need to evaluate first the classical limit of the discriminant. Note that the discriminant of a polynomial can be evaluated up to a multiplicative constant by *

$$
\Delta[P]=\prod_{x_{i} \in S} P\left(x_{i}\right)
$$

where $x_{i} \in S$ are the critical points of $P, \partial_{x} P\left(x_{i}\right)=0$. Differentiating the polynomial in (3.3) with respect to $x$ and equating to zero we have

$$
P^{\prime}=2 \mathcal{C}(x) \mathcal{C}(x)^{\prime}-\varepsilon G\left(x, m_{i}\right)^{\prime}=0,
$$

${ }^{*}$ The discriminant of a degree $n$ curve is a polynomial in its roots of degree $n(n-1)$ that vanishes when any two roots of the curve coincide. It is easy to see that (3.4) satisfies these. 
where we denoted $\varepsilon=\Lambda_{N_{f}}^{2 h-2 N_{f} \mu}$. The roots of equation (3.5) are $\left\{r_{i}=a_{i}+\varepsilon\right.$ corrections $\}$ and $\left\{s_{i}=b_{i}+\varepsilon\right.$ corrections $\}$ where $a_{i}$ and $b_{i}$ are the roots of $\mathcal{C}(x)$ and $\mathcal{C}(x)^{\prime}$, respectively. According to (3.4)

$$
\Delta[P]=\prod_{i} P\left(r_{i}\right) \prod_{j} P\left(s_{j}\right)
$$

In order to analyze the classical limit we have to evaluate (3.6) as $\varepsilon \rightarrow 0$. Consider the first product in (3.6) and let us prove that to lowest order in $\varepsilon$

$$
\prod_{i} P\left(r_{i}\right)=\varepsilon^{h_{g}} \prod_{i} G\left(a_{i}, m_{j}\right)
$$

which is the contribution of the $\varepsilon G$ term in $P$. We have to show that the $\mathcal{C}(x)$ contribution is of higher order in $\varepsilon$. Suppose $a_{i}$ is a root of $\mathcal{C}(x)$ of multiplicity $n$, then it follows from (3.5) that

$$
r_{i}=a_{i}+c_{i} \varepsilon^{\frac{1}{2 n-1}}+\ldots .
$$

Thus the contribution of $\mathcal{C}\left(r_{i}\right)^{2}$ is of order $\frac{2 n}{2 n-1}>1$ in $\varepsilon$ which is higher than the contribution of the $\varepsilon G$ term. In order to get the lowest order in $\varepsilon$ we evaluate the second product in (3.6) at the roots $b_{i}$, which yields using (3.4)

$$
\prod_{j} P\left(b_{j}\right)=\Delta[\mathcal{C}]^{2}
$$

where $\Delta[\mathcal{C}]$, given by $(2.23)$ is the discriminant of the classical curve (2.8). Thus, (3.7) and (3.9) yield

$$
\Delta[P]=\varepsilon^{h_{g}} \Delta[\mathcal{C}]^{2} \prod_{i} G\left(a_{i}, m_{j}\right) .
$$

A comparison of (3.10) to (2.22),(2.23) and (2.24) yields

$$
G\left(x, m_{i}\right)=\operatorname{det}\left(x^{2}-M^{2}\right)
$$

which completes the proof.

The meromorphic one-form $\lambda$, satisfying (2.17), takes the form

$$
\lambda=\frac{x d x}{2 \pi i y}\left(\frac{\mathcal{C} G^{\prime}}{2 G}-\mathcal{C}^{\prime}\right),
$$

for $2 N_{f} \mu<h$. The fact that the residues of $\lambda$ satisfy (2.18) is a corollary of the residue calculation that will be presented in the next section. This provides a consistency check on our result. 


\section{The quantum moduli space for $N_{f} \geq N_{c}-l-2$}

\subsection{The case $N_{f}=N_{c}-l-2$}

The most general curve consistent with $R$-symmetry, instanton corrections and classical singularity is

$$
y^{2}=\mathcal{C}^{2}(x)-\Lambda_{N_{f}}^{2 l}\left(x^{d} \operatorname{det}\left(x^{2} I_{f}-M^{2}\right)+a \mathcal{C}(x)\right)+b \Lambda^{4 l},
$$

where $a$ and $b$ are constant coefficients. Since the one instanton correction $\Lambda^{h}$ has $R$ charge $h$, the curve (4.1) gets contributions also from a two instanton process of the form $\Lambda^{2 h}$. The structure of the correction $\Lambda^{h} \mathcal{C}(x)$ in (4.1) is determined such that it vanishes for $x=a_{i}$ as required, via the analysis of the previous section, by comparing (3.10) with $(2.24)$.

The curve (4.1) can be written in a form suitable for generalization to $N_{f}>N_{c}-l-2$ :

$$
y^{2}=F^{2}-H,
$$

where

$$
\begin{aligned}
& F=\mathcal{C}+\alpha \Lambda^{h} \\
& H=\Lambda^{h}\left(x^{d} \operatorname{det}\left(x^{2} I_{f}-M^{2}\right)+\beta \Lambda^{h}\right)
\end{aligned}
$$

with $\alpha$ and $\beta$ constants.

The meromorphic one-form $\lambda(2.17)$ reads in this case

$$
\lambda=\frac{x d x}{2 \pi i y}\left(\frac{F H^{\prime}}{2 H}-F^{\prime}\right) .
$$

The residue formula (2.18) can be used in order to determine the coefficient $\beta$, as we will show now. This will be an example of a powerful constraint which will be much used in the sequel.

Let us consider the case of equal bare masses $m_{i}=m$. The zeros of $H$ in (4.3) read

$$
x_{i}=-m+e^{\frac{2 \pi i}{N_{f}}} \beta^{\frac{1}{N_{f}}} \Lambda, \quad i=1, \ldots, N_{f}-1 .
$$

The residue of $\lambda$ in (4.4) at the root $x_{0}$ of (4.5) is

$$
2 \pi i \underset{x=x_{0}}{\operatorname{res}}(\lambda)=\frac{m-\beta}{2},
$$


thus (2.18) implies $\beta=0$. The residues of $\lambda$ at zeros of $y$ vanish. This is easily seen by differentiating (4.2) with respect to $x$, which together with (4.2) at $y=0$ yield

$$
\left(\frac{F H^{\prime}}{2 H}-F^{\prime}\right)_{y=0}=0 .
$$

Thus, we verified that the residue formula (2.18) is satisfied completely by $\lambda$ of (4.4). Note that we are still left with one undetermined constant coefficient $\alpha$. This constant may br determined by global symmetry arguments. We choose to absord this constant by redefining $s_{2 l}$.

Compatibility with the hyper-elliptic curves for $N_{c}>N_{f}$ which will be discussed in the next section implies that $\alpha=\frac{1}{4}$ for $N_{c}>2$, however, we do not have a full proof for that.

Thus, we suggest that the family of curves describing the quantum moduli space of vacua for the $N_{f}=N_{c}-l-2$ is

$$
y^{2}=\mathcal{C}^{2}(x)-\Lambda^{2 l} x^{d} \operatorname{det}\left(x^{2} I_{f}-M^{2}\right) .
$$

\subsection{The quantum moduli space for $N_{f}>N_{c}-l-2$}

When the number of flavors is increased the curves describing the quantum moduli spaces may get contributions from higher multi-instanton processes. This increases the number of terms in the polynomials describing the curves that should be determined. In this section we study the general $N_{f}>N_{c}$ cases. We parameterize the curves and determine there structure up to a certain number of unknown constant coefficients. Global symmetry considerations may suggest a fixed value for these constants. We reabsorb the constants in a redefinition of the symmetric functions $s_{2 k}$.

The general structure of the family of curves describing the quantum moduli space of vacua when $N_{f}>N_{c}-l-2$ is encoded in the following claim.

Claim: The curve describing the quantum moduli space with gauge group $S O\left(N_{c}\right)$ and $N_{f}>N_{c}-l-2$ flavors is:

$$
y^{2}=\left(\mathcal{C}(x)+\Lambda^{2\left(N_{c}-N_{f}-2\right)} P\right)^{2}-\Lambda^{2\left(N_{c}-N_{f}-2\right)} x^{d} \operatorname{det}\left(x^{2} I_{f}-M^{2}\right)
$$

where $P\left(x, m_{i}, \Lambda\right)$ is a polynomial of degree $h+2\left(N_{f}-N_{c}+2\right)$ in $x, m_{i}$ and is independent of the moduli $s_{k}$.

Proof: Consider the most general hyper-elliptic curve. Using $\partial_{2 k} \partial_{2 l} \lambda=\partial_{2 l} \partial_{2 k} \lambda$ where $\partial_{k} \equiv \frac{\partial}{\partial s_{k}}$ together with (2.17) yields

$$
x^{-2 k} \partial_{2 l} y^{2}=x^{-2 l} \partial_{2 k} y^{2}
$$


Equation (4.10) implies that $y^{2}$ depends on the moduli $s_{k}$ only via $\mathcal{C}(x)$. Thus, $y^{2}\left(s_{k}\right)=$ $y^{2}(\mathcal{C}(x))$. Moreover, $R$ charge symmetry implies that only terms up to quadratic in $\mathcal{C}(x)$ can appear:

$$
y^{2}=\mathcal{C}^{2} g_{0}+\mathcal{C} g_{1}\left(x, m_{i}, \Lambda\right)+g_{2}\left(x, m_{i}, \Lambda\right),
$$

where $g_{0}$ is a polynomial of degree 0 in $x$, namely a constant ${ }^{*}, g_{1}, g_{2}$ are polynomials in $x$ of degree $h_{g}$ and $2 h_{g}$, respectively and are independent of the moduli $s_{k}$. The classical limit fixes $g_{0}=1$. The curve (4.11) may be recast in the form

$$
y^{2}=\left(\mathcal{C}+\Lambda^{2\left(N_{c}-N_{f}-2\right)} P\right)^{2}-\Lambda^{2\left(N_{c}-N_{f}-2\right)} G,
$$

with

$$
G\left(x, m_{i}, \Lambda\right)=x^{d} \operatorname{det}\left(x^{2} I_{f}-M^{2}\right)+\sum_{k=1}^{\left[\frac{2 N_{c}}{2\left(N_{c}-N_{f}-2\right)}\right]-1} \Lambda^{2 k\left(N_{c}-N_{f}-2\right)} n_{k}(x, m),
$$

where we used $R$ charge considerations and the form of instanton corrections. The first term in (4.13) has been deduced from the structure of the classical singularity in section 3. The other terms are arranged according to the order of multi-instanton contribution. $n_{k}$ is a polynomial of degree $(k+1) N_{f}-2 k N_{c}$ and $\left[\frac{2 N_{c}}{2 N_{c}-N_{f}}\right]$ denotes its integer value. $P\left(x, m_{i}, \Lambda\right)$ is a polynomial of degree $N_{f}$ in $x, m_{i}$.

We will now use the residue formula (2.18) in order to determine the form of $G(x)$ in (4.12). The meromorphic one-form $\lambda(2.17)$ associated with (4.12) is

$$
\lambda=\frac{x d x}{2 \pi i y}\left(\frac{F G^{\prime}}{2 G}-F^{\prime}\right),
$$

where, as before, prime denotes derivative with respect to $x$ and $F=\mathcal{C}+\Lambda^{2\left(N_{c}-N_{f}-2\right)} P$.

Consider the zeros $x_{i}$ of $G(x)$ where $y\left(x_{i}\right) \neq 0$ and let us evaluate $\operatorname{res}(\lambda)$ at these points. Since $F / y= \pm 1$ at $x_{i}$ and $G^{\prime} / G$ equal the order $n$ of the root $x_{i}$ we get

$$
2 \pi i \underset{x=x_{i}}{\operatorname{res}}(\lambda)= \pm \frac{n}{2} x_{i}
$$

Comparing (4.15) to (2.18) we conclude that the roots of $G(x)$ do not receive quantum corrections, thus $n_{k}=0$ in (4.13). This completes the proof of the claim.

In order to fully construct the curve we still have to determine the polynomial $P$. Studies of the structure of the singularities, compatibility with the global symmetries may determine $P$. We will absorb $P$ into a redefinition of $G(x)$ which is a linear transformation

${ }^{*}$ As we will discuss in the sequel, when $N_{f}=N_{c}-2, g_{0}$ may be a modular form $g_{0}(q)$. 
on $s_{2 k}$ which depends on the masses $m_{i}$ and the dynamical scale $\Lambda$ but does not change their classical limit. Thus, we suggest that the familiy of curves describing the quantum moduli space of vacua for $N_{f}>N_{c}-l-2$ is

$$
y^{2}=\mathcal{C}^{2}(x)-\Lambda^{2\left(N_{c}-N_{f}-2\right)} x^{d} \operatorname{det}\left(x^{2} I_{f}-M^{2}\right)
$$

\section{The quantum moduli space for $N_{f}=N_{c}-2$}

\subsection{The general case}

When $N_{f}=N_{c}-2$ with the bare masses and all but one of the moduli set to zero we get conformally invariant theories. In these cases the classical relations (2.4) and (2.5) are expected to be valid quantum mechanically. Thus,

$$
a_{D}^{i}=\tau_{i j} a^{j}
$$

where $\tau_{i j}$ is the matrix of theta angles and coupling constants of the theory. The classical and quantum moduli spaces are identical and are described by a hyper-elliptic curve with period matrix $\tau_{i j}$ and periods as in (2.16) with $a^{i}, a_{D}^{i}$ satisfying (2.5).

The structure of the family of curves describing the moduli space of vacua for $N_{f}=$ $N_{c}-2$ is encoded in the following:

Claim: The curve for the quantum moduli space for $S O\left(N_{c}\right)$ gauge group with $N_{f}=$ $N_{c}-2$ is:

$$
y^{2}=\left[\mathcal{C}\left(x, l(q) s_{k}\right)+P(x, l(q) m, q)\right]^{2}-L(q) \operatorname{det}\left(x^{2} I_{f}-l(q) M^{2}\right) .
$$

$l(q)$ is a modular form satisfying $l(q) \rightarrow 1$ as $q \rightarrow 0 . \quad P(x, l(q) m, q)$ is a modular form satisfying $P \propto q$ as $q \rightarrow 0$ and a polynomial of degree $N_{c}$ in $x$ which is independent of the moduli $s_{k}$. $L(q)$ is a modular form of weight zero satisfying $L(q) \propto q+O\left(q^{2}\right)$.

Proof: The proof is similar to that of section 5.3. The main difference is that the dynamically generated scale $\Lambda$ is replaced by $q$ as defined in (2.28). The structure of the first term in (5.2) is deduced following the same argument as in equations (4.10) (4.12) together with $R$-symmetry. The factor $g_{0}$ of (4.11) gets contributions both from $\mathcal{C}\left(x, l(q) s_{k}\right)$ and $P(x, l(q) m, q)$. The modification of all the moduli $s_{k}$ by the same modular form $l(q)$ is consistent with $R$-symmetry as well as (4.10) and (4.11).

The structure of the second term follows from the analysis of the residues (4.15) of the meromorphic one-form $\lambda(4.14)$, which now gets a pre-factor $\frac{1}{l(q)}$. This implies that $m_{i}$ must be rescaled by $l(q)$ in order to ensure that the residues of $\lambda$ be independent of $\tau$. The 
behavior of $L(q)$ as $q \rightarrow 0$ is implied by the matching condition (2.28) when integrating a massive quark. It is computed by requiring the curve to have the classical period matrix.

$P$ may determined by global symmetries and singularity structure. We choose to absorb it in a redefinition of $\mathcal{C}(x)$. This amounts to shifts in $s_{2 k}$ by functions which depend on $q$ and $m_{i}$ but do not affect their classical limit. Thus we propose the curve

$$
y^{2}=\mathcal{C}^{2}\left(x, l(q) s_{k}\right)-L(q) x^{d} \operatorname{det}\left(x^{2} I_{f}-l(q) M^{2}\right) .
$$

Consider now the curve (5.3) with the masses being set to zero and let us use the complex line $l$ defined by $s_{i}=0, i \neq h, s_{h}=s$. This curve contains only one scale therefore it is scale invariant. $L(q)$ will be determined by requiring the curve to have the classical period matrix.

\section{Discussion and conclusions}

In this paper we constructed the hyper-elliptic curves which describe the quantum moduli spaces of vacua of $N=2$ supersymmetric $S O\left(N_{c}\right)$ gauge theories with $N_{f}$ flavors of quarks in the vector representation. We showed that the curves for $N_{f}<N_{c}-l-2$ are completely determined by $R$-symmetry, the form of instanton corrections and the requirement for the correct classical singularity structure. The compatibility of the residue calculations with the BPS formula as well as the correct weak coupling monodromy provide further support to the results.

Weak coupling monodromies are computed along the same lines of reference [7] for all the curves and can be shown to coincide with what is expected on physical grounds, thus providing a check on the results. We left the calculation of the strong coupling monodromies for the future. This will clearly be needed in order to extract the physics of these theories. Along the way we derived the exact metrics on the quantum moduli spaces as well as the exact spectrum of stable massive states.

The ability to extract exact results in the theories that were studied in this paper points to an underlying integrable structure [16]. In particular, one expects that the prepotential will be related to a $\tau$ function of some integrable hierarchy and that the variety describing the quantum moduli space of vacua will arise as a solution to a non-linear integrable equation of the hierarchy. Revealing these structures may provide us with

powerful computational tools for these four-dimensional models. See for example [18]. An Example of a strong consistency check would be the calculation of the Witten index from the curves and then compare it to the expectation from weak coupling behavior for 
large masses of the adjoint and the quark fields.

In a recent paper Martinec and Warner showed that the Riemann surfaces which describe the moduli space of quantum vacua for $N=2$ supersymmetric Yang-Mills theories with a simple gauge group $G$ is described by spectral curves of the periodic Toda lattice for the dual group. Their solution coincides with the known curves so far and provides new solutions for the unknown cases. The curves read

$$
\begin{aligned}
A_{l} & : z+\frac{\mu}{z}+\mathcal{C}(x)=0 \\
B_{l} & : x\left(z+\frac{\mu}{z}\right)+\mathcal{C}(x)=0, \\
C_{l} & :\left(z-\frac{\mu}{z}\right)^{2}+\mathcal{C}(x)=0 \\
D_{l} & : x^{2}\left(z+\frac{\mu}{z}\right)+\mathcal{C}(x)=0
\end{aligned}
$$

and similar expressions for the exceptional groups. There exist simple tranformations between the forms of $A_{l}, B_{l}, D_{l}$ and the known curves in the literature [17]. There is no known expression for the $C_{l}$ groups in terms of hyper-elliptic curves.

The parameter $\mu$ for $N_{f}=0$ in equation (6.1) is $\mu=\Lambda^{2 h}$. $h$ being the second Casimir of the group. Henceforth we will denote the Dynkin index of a representation by $T(r)$. The previous works on $A_{l}$ theories with $N_{f}$ flavors in the fundumental representation $[7,8]$ give $\mu=\Lambda^{2 h-2 N_{f} T(r)} \operatorname{det}\left(x I_{f}+M\right)$ for any flavor. (The masses are rescaled by a factor which depend on $\tau$ for the finite theories.) This work suggests that for $B_{l}$ and $D_{l}$ groups with $N_{f}$ flavors in the vector representation $\mu=\Lambda^{2 h-2 N_{f} T(r)} \operatorname{det}\left(x^{2} I_{f}-M^{2}\right)$. On the basis of this we conjecture that to any $N_{f}$ for $C_{l}$ groups

$$
\mu=\Lambda^{2 h-2 N_{f} T(r)} \operatorname{det}\left(x^{2} I_{f}+M^{2}\right) .
$$

Similar formulae are argued for the exceptional groups. $\mu=\Lambda^{2 h-2 N_{f} T(r)} \operatorname{det}\left(x^{\alpha}-M^{\alpha}\right)$, Where $\alpha$ is an integer which depends on the group and on the representation.

\section{Acknowledgements}

I would like to thank K. Intriligator and E. Witten for helpful discussions. 


\section{References}

[1] N. Seiberg and E. Witten, "Monopole Condensation, And Confinement In $N=2$ Supersymmetric Yang-Mills Theory," hep-th/9407087, Nucl. Phys. B426 (1994) 19, Erratumibid.B430:485-486,1994.

[2] N. Seiberg and E. Witten, "Monopoles, Duality and Chiral Symmetry Breaking in $N=2$ Supersymmetric QCD," hep-th/9408099, Nucl. Phys. B431 (1994) 484.

[3] A. Klemm, W. Lerche, S. Yankielowicz, S. Theisen, "Simple Singularities and $N=2 \mathrm{Su}-$ persymmetric Yang-Mills Theory," hep-th/9411048, Phys. Lett. 344B (1995) 169;

"On the Monodromies of $N=2$ Supersymmetric Yang-Mills Theory," hep-th/9412158, Talk given at Workshop on Physics from the Planck Scale to Electro-magnetic Scale, Warsaw, Poland, Sep 21-24, 1994 and at 28th International Symposium on Particle Theory, Wendisch-Rietz, Germany, 30 Aug - 3 Sep 1994.

[4] P. C. Argyres and A. E. Faraggi, "The Vacuum Structure and Spectrum of $N=2$ Supersymmetric SU(N) Gauge Theory," hep-th/9411057, IASSNS-HEP-94-94.

[5] M. R. Douglas and S. H. Shenker, "Dynamics of $S U(N)$ Supersymmetric Gauge Theory," hep-th/9503163, RU-95-12.

[6] P. C. Argyres and M. R. Douglas, "New Phenomena in $S U(3)$ Supersymmetric Gauge Theory," hep-th/9505062, IASSNS-HEP-95/31, RU-95-28.

[7] A. Hanany and Y. Oz, "On the Quantum Moduli Space of Vacua of $N=2$ Super-symmetric $S U\left(N_{c}\right)$ Gauge Theories," hep-th/9505075, TAUP-2248-95, WIS-95/19/May-PH

[8] P. C. Argyres, M. R. Plesser and A. Shapere, "The Coulomb Phase of $N=2$ Supersymmetric QCD," hep-th/9505100, Phys. Rev. Lett. 75 (1995) 1699.

[9] J. A. Minahan and D. Nemeschansky, "Hyperelliptic curves for Supersymmetric YangMills," hep-th/9507032,USC-95/019, CERN-TH. 95-167.

[10] U. H. Danielsson and B. Sundborg, "The Moduli Space and Monodromies of $N=2$ Supersymmetric $S O(2 r+1)$ Yang-Mills Theory," hep-th/9504102, USITP-95-06, UUITP-4/95.

[11] A. Brandhuber and K. Landsteiner, "On the Monodromies of $N=2$ Supersymmetric Yang-Mills Theory with Gauge Group $S O(2 n)$," hep-th/9507008, CERN-TH/95-180.

[12] E. Witten and D. Olive, "Super-symmetry Algebras that include

[13] N. Seiberg, "Super-symmetry And Non-perturbative Beta Functions," Phys. Lett. 206B (1988) 75 .

[14] D. Finnell, P. Pouliot, "Instanton Calculations Versus Exact Results in Four Dimensional Susy Gauge Theories," hep-th/9503115, SLAC-PUB-95-6768. 
[15] N. Seiberg, "Electric - Magnetic Duality in Supersymmetric Non-Abelian Gauge Theories," hep-th/9411149, Nucl. Phys. B435 (1995) 129.

[16] A. Gorsky, I. Krichever, A. Marshakov, A. Mironov and A. Morozov, "Integrability and Seiberg-Witten Exact Solution," hep-th/9505035, UUITP-6-96, ITEP-M3/95, FIAN/TD9-95.

[17] E. Martinec and N. Warner, "Integrable systems and supersymmetric gauge theory," hepth/9509161,EFI-95-61, USC-95/025.

[18] T. Nakatsu and K. Takasaki, "Whitham-Toda Hierarchy and $N=2$ Supersymmetric YangMills Theory," hep-th/9509162, KUCP-0083. 